\title{
ON THE CIRCUITS OF PLANE CURVES*
}

\section{CHARLOTTE ANGAS SCOTT}

1. The nature of the individual circuits (or complete branches) that make up a curve of order $n$ has not received very much attention. Von STAUdT (1847) distinguished between odd and even circuits; MoEbius (1852, Ueber die Grundformen der Linien der dritter Ordnung) by projection on to a sphere from the centre, brought out even more clearly the distinction, since the odd circuit is represented on the sphere by a single line, the even circuit by two distinct lines. Cayley (1865, On Quartic Curves, Collected Papers, vol. 5, op. 361) applied the Moebius projection to the quartic, and thus proved that not only the individual circuits of any non-singular quartic, but all the circuits at once, can be projected into the finite part of the plane. He pointed out that this conclusion does not hold as regards the non-singular sextic; there exists such a curve, composed of a single circuit, which cannot be projected into the finite part of the plane. On a sphere, this circuit is not confined to one hemisphere. In the concluding paragraph the remark is made that a quartic with one node may consist of two odd circuits; such a quartic is not the projection of any finite curve. Clifford (1870, Synthetic Proof of Miquel's Theorem) mentioned another sextic that is not confined to one hemisphere. ZeUthen, in his classical discussion of quartic curves (1874, Mathematische Annalen, vol. 7) emphasized the distinction between odd and even circuits, and proved the important theorem that in one, and only one, of the two regions $\dagger$ into which a plane is divided by a nonsingular even circuit, odd circuits can lie. He showed (p. 426) that a quartic can be composed of a single even circuit, cutting itself twice, of such a form that every straight line meets it in at least two real points. There may be an additional double point of any kind, or the curve may have a simple circuit, necessarily a simple oval. A quartic of this character, with $p=0$, can be obtained by quadric inversion from a conic which separates one of the three fundamental points from the other two. If such a circuit be projected on to the sphere, it will be found that the two lines by which it is represented interlace.

2. The theorems mentioned suggest that there is a general theorem as to the existence of circuits that cannot be projected into the finite part of the plane,

* Presented to the Society April 26, 1902. Received for publication May 29, 1902.

tOn a sphere, there are three regions. 
that is, circuits some of whose intersections with every straight line are real. Let the maximum number of points in which a circuit is met by a straight line be called its order; let the minimum number be called the index of the circuit; it is obvious that

and that

$$
\text { order }- \text { index } \equiv 0(\bmod 2) \text {, }
$$

$$
\text { order }- \text { index } \geqq 2 \text {. }
$$

The general theorem can be stated in the form :

For every order $n$ there exist curves, $p=0$ or 1 , formed of a single circuit of index $n-2$.

The curves $p=1$ may have also another circuit, without point- or line-singularities.

Further, for every order $n$ there exist curves, $p=0$ or 1 , formed of a single circuit of index $n-2 r$, where $r$ may have any value from 1 to $n / 2$ if $n$ be even, from 1 to $(n-1) / 2$ if $n$ be odd.

3. As a preliminary, in order to form some idea of the appearance of such a curve, consider a curve of order $n(p=n-2)$ composed of $n-2$ odd circuits through $O$, namely

$$
\left(x^{2}+y^{2}\right) \prod_{i=1}^{i=n-2} l_{i}+\prod_{i=1}^{i=n-2} t_{i}=0
$$

where the $l$ 's and $t$ 's represent real lines through $O$, alternating. Let these odd circuits be drawn so that they do not all pass through $O$, as in Fig. 1, for which $n=5$. If now the first circuit be linked with the second, and the second with the third, as indicated in Fig. 1', a single circuit of index 3 is formed with the help of two new double points $B, C$.

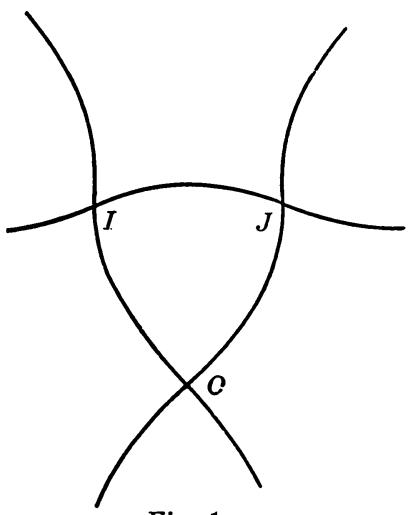

Fig. 1

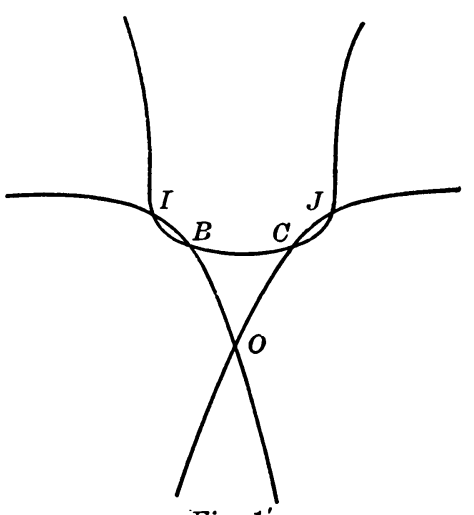

Fig. 1'

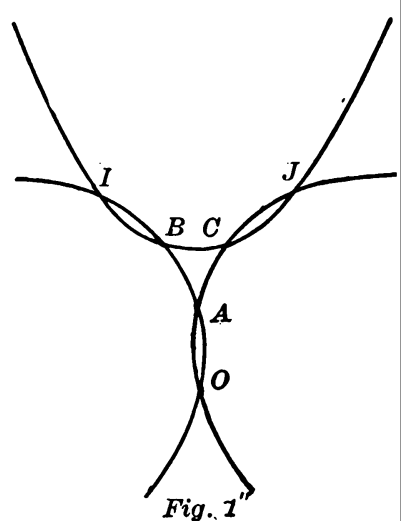

Fig. 1"

Similarly the $n-2$ circuits, linked by means of $n-3$ new double points, become a single circuit of index $n-2$. If this process of deformation is 
admissible, the resulting curve is a $C_{m}, p=1$, composed of a single circuit, for which

$$
\text { order }- \text { index }=2 \text {. }
$$

A different linking of the circuits is shown in Fig. $1^{\prime \prime}$, accomplished by means of three new double points ; the curve is a quintic, $p=0$.

4. The rigorous proof of the theorem is obtained by means of Cremona transformations. By this means also the validity of this process of linking the circuits is established.

When an oval is subjected to quadric inversion, its position with respect to the fundamental triangle $O I J$ determines the appearance of the resulting circuit. If the oval does not meet any of the fundamental lines, it transforms into an oval, with or without indentations; if it crosses any of the fundamental lines, but in such a manner as not to enclose any of the fundamental points, it becomes a branch cutting itself, but still such that it can be projected into the finite part of the plane. If however the oval surrounds one of the fundamental points, $O$, it necessarily meets in at least two real points all conics through $O I J$, and since these correspond to the straight lines of the transformed plane, the resulting circuit is of index 2, of the type of the Zeuthen quartic circuit. A similar result follows if the oval surrounds two of the fundamental points.

An oval that passes through one of the fundamental points transforms into an odd circuit, of index 1 , passing through the two fundamental points whose correspondents are the two fundamental lines that meet in the point through which the oval passes.

The quintic of which the existence was suggested by the process of deformation can actually be obtained by inversion from a quartic. Let $u=0$ be a quartic consisting of three ovals; let $O, I, J$ be points on these, chosen for the

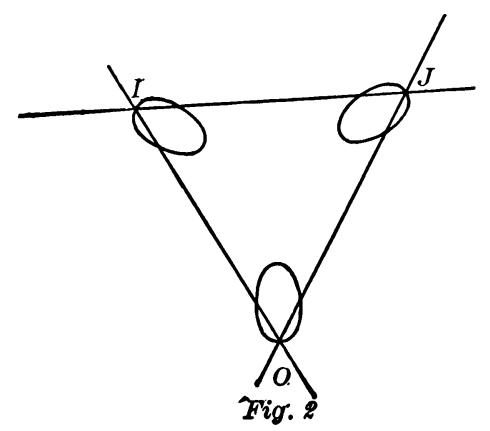

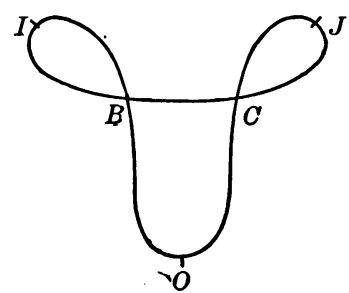

Fig. $\mathscr{2}^{\prime}$

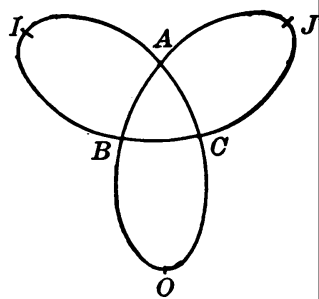

Fig. $2^{\prime \prime}$

sake of simplicity so that the quartic touches a conic $v=0$ at these points (Fig. 2). The quartics $u+\lambda v^{2}=0$, where $\lambda$ is a variable parameter, form a system through $O I J$, lying outside $u$ if the sign of $\lambda$ is properly chosen. These invert 
into a system of quintics, composed of three odd circuits intersecting at $O, I, J$. If $u, v$ are chosen so that the whole figure has a single axis of symmetry through $O$, the two double points $B$ and $C$ (Fig. $2^{\prime}$ ) present themselves for the same value of $\lambda$, and the circuits are linked as in Fig. $\mathbf{1}^{\prime}$. If there is triangular symmetry, the three double points $A, B, C$ appear for some one value of $\lambda$, (Fig. 2"), and the transformed curve is as shown in Fig. 1".

In the general Cremona transformation the straight lines of the transformed plane are derived from rational curves passing through the fundamental points in the original plane, forming a Cremona net. If the curve to be transformed entirely surrounds a fundamental point of odd order, it is cut in at least two real points by every curve of the Cremona net; the transformed curve consequently meets every straight line in its plane in at least two real points, and is therefore of index 2. The number of fundamental points inside any one loop, link, or oval, makes no difference, so long as there are fundamental points of odd order outside also. Similarly the presence of a fundamental point of odd order on the boundary of a loop, link, or oval ensures one real intersection other than the fundamental point itself.

5. The simplest type of Cremona net is composed of curves of order $q$ with a fixed $(q-1)$-point and $2(q-1)$ fixed simple points. By means of this net the theorem can be proved. It will be shown in the first place that for every order $m$ there exists a curve with a multiple point of order $m-3$ and $2(m-3)$ links, if $p=1$, but with $2(m-3)+1$ links if $p=0$. Such curves are familiar among quartics, as shown in Fig. $3(p=0)$ and Fig. $3^{\prime}(p=1)$.

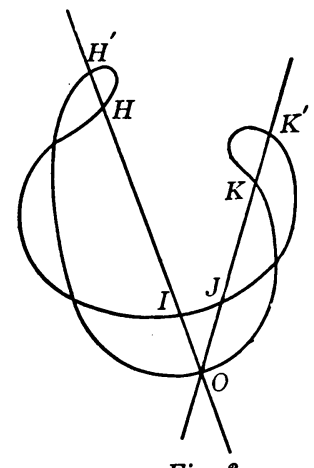

Fig. $s$

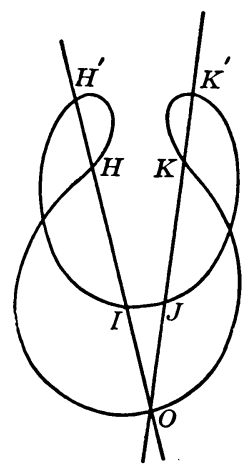

Fig. $\mathbf{s}^{\prime}$

A point $O$ is chosen on the part of the curve indicated in the figure, and from $O$ two lines are drawn to meet the final loops of the curve in real points, which is certainly possible for these quartics ; these lines meet the quartic again at $I$, $J$. By means of a quadric inversion with $O I J$ as fundamental points the curve is transformed into a quintic, with a double point at $O$ since the line $I J$ meets 
the quartic again in two points. The pairs of points $H, H^{\prime} ; K, K^{\prime}$ give rise to new double points at $I, J$, and the resulting quintic is of the desired type. (Figs. 4, 4.')

Two lines can be drawn through $O$ to meet the loops in real points, $H, H^{\prime}, K, K^{\prime}$; these lines meet the base of the curve each in one point, necessarily real, and therefore available as fundamental points $I, J$. The line $I J$ meets the quintic again in three points, hence the transformed curve, a sextic,

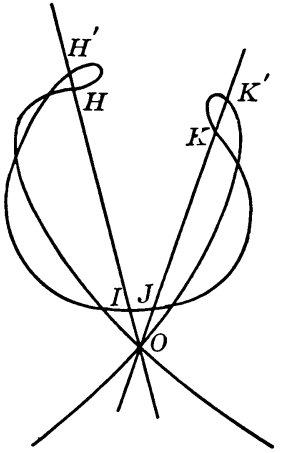

Fig. 4

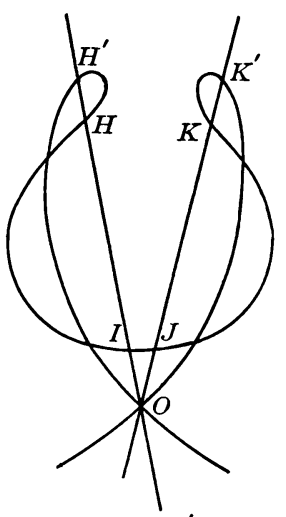

Fig. 4'

has a triple point at $O$; and as before the points $H H^{\prime}, K K^{\prime}$ give rise to double points at $I, J$, and the two series of links in the curve are increased each by unity. The curve is a $C_{6}$, with a 3-point, and $2.3+1$ links if $p=0,2.3$ links if $p=1$.

In precisely the same manner we obtain from a $C_{m}$ with an $(m-3)$-point and $2(m-3)+1$ [or $2(m-3)$ ] links a curve of order $2 m-(m-3)-2$, that is, $m+1$, with, at $O$, a point of order $m-1-1$, that is, $m+1-3$, and $2(m-3)+1+2$, that is $2\{(m+1)-3\}+1$ [or $2(m-3)+2$, that is, $2\{(m+1)-3\}]$ links. It is not necessary to consider whether the branches that form the multiple point at $O$ are all real; this is immaterial. What is essential is that the links be all real, and this is shown by the manner in which the curve is produced.

6. We now apply a Cremona transformation of the kind mentioned, choosing $q$ so that there shall be a fundamental point available for the interior of every link. This requires $q=m-2$. The curve $C_{m}$ with an $(m-3)$-point and $2(m-3)$ links is transformed by means of curves of order $m-2$ with a fixed $(m-3)$-point and $2(m-3)$ fixed simple points. The transformed curve is therefore of order

$$
n=m(m-2)-(m-3)^{2}=4 m-9,
$$

and the index, to which every link contributes 2 , is at least $2 \times 2(m-3)$. 
Since however $4(m-3)=4 m-9-3$, which gives for order - index the odd value 3 , the index must be greater than $4(m-3)$. It cannot be greater than $4 m-9-2$, hence

$$
\text { index }=4 m-11=n-2 \text {. }
$$

This proves the theorem for every $n$ of the form $4 m-9$, that is, $4 k-1$; for the intermediate values all that is necessary is to place $1,2,3$ of the fundamental points on the boundaries of links instead of inside. Every such placing of a fundamental point diminishes both order and index by unity, and thus leaves undisturbed the relation, now proved for all values of $n$,

$$
\text { order }- \text { index }=2 .
$$

The fundamental curves for the Cremona net here used are

(i) $2(m-3)$ straight lines joining $O$ to the other fundamental points, singly ; (ii) a $C_{m-3}$ with an $(m-4)$-point at $O$, passing singly through the other fundamental points.

Since each of the straight lines (i) meets $C_{m}$ in 3 points that do not lie at the fixed points, the $C_{n}$ has $2(m-3)$ triple points if $n=4 m-9$, but $1,2,3$ of these are replaced by double points if $n=4 m-9$ diminished by $1,2,3$. The fundamental $C_{m-3}$ gives rise to a multiple point of order $4(m-3)$ on $C_{n}$, diminished by 1,2, 3 for the special cases named; the order of this point is therefore in all cases $n-3$. There are also the unaltered double points of the original curve. These make up exactly the right number for the $C_{n}$ with $p=0$ or 1 .

If now $h$ of the fundamental points are placed outside the links instead of inside, the order is not affected, but the index is diminished by $2 h$. In this way, for the curve of order $4 m-9$ the index can be made as low as 1 , instead of $4 m-11$. For the intervening orders the lowest index, 1 or 0 , is obtained by placing the last 1, 2, 3 fundamental points on the base of the curve.

This completes the proof of the theorem.

7. For greater values of $p$ it can be shown that the index can attain to certain specified values, but proof is still lacking that these are the highest possible values * for any given $n$ and $p$. If $p$ be of the form $4 t-2,4 t-1,4 t, 4 t+1$, then for every order $n$, where $n \geqq 2(t+1)$, there exists a curve composed of a single circuit for which

$$
\text { order }- \text { index }=2(t+1) .
$$

For the proof of this, the first step is to demonstrate the existence of curves of every order $m$ of the type shown in Figs. 5 and $5^{\prime}$, each with an $(m-3)$. point at $O$. For any such curve

\footnotetext{
* In fact, they are not the highest values, as witness the sextic, $n=6, p=10$, index $=2$.
} 


$$
p=\frac{1}{2}(m-1)(m-2)-\frac{1}{2}(m-3)(m-4)
$$

diminished or not by unity; that is, $p=2 m-6$, Fig. 5 , and $p=2 m-5$, Fig. 5'.

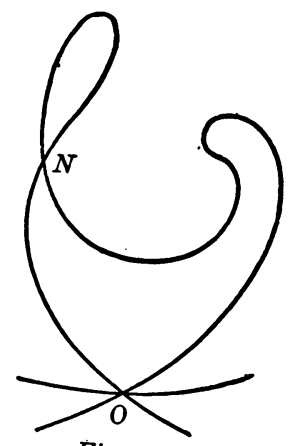

Fig. 5

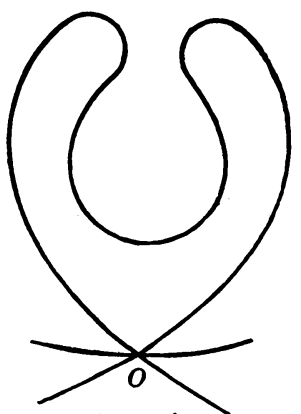

Fig. $5^{\prime}$

If the existence of such a curve, $u_{m}=0$, be assumed for any value of $m$, it follows at once for the value $m+2$.

(1) Let $m$ be even, the desired $C_{m+2}$ is given by

$$
f_{2} u_{m}=\lambda F_{m} \phi_{2}
$$

with a sufficiently small value for $\lambda$, where $f_{2}$ is an imaginary line-pair through $O, F_{m}$ a set of $m$ imaginary lines through $O$, and $\phi_{2}$ a pair of imaginary lines, not through $O$, but intersecting at $N$ if this other node exists.

(2) Let $m$ be odd ; then with the same interpretation for $f, F$, and $\phi$ the conditions are fulfilled by

$$
f_{2} u_{m}=\lambda F_{m-1} z \phi_{2},
$$

where $z=0$ is the straight line at infinity.

This proves that if curves of the kind indicated exist for order $m$, they exist for order $m+2$. They do exist for $m=4$; and the equation

$$
y u_{4}=\lambda F_{2} z \phi_{2}
$$

proves that they do exist for $m=5$, and consequently for every value of $m$. Any such curve may have also certainly one oval or even two, possibly more.

If we now transform these curves by quadric inversion, exactly as for the case of $p=0$ or 1 , we obtain from each a series of curves, as shown in Fig. 6, where curves of the same order are represented in the columns, and curves with the same $p$ in the rows.*

* These diagrams show the nodes and links accurately as to number and general situation; they are not intended to show directions to infinity or inflexions. In fact, some inflexions not aotually existing on the ourves have been inserted on the links, for olearness in drawing. 


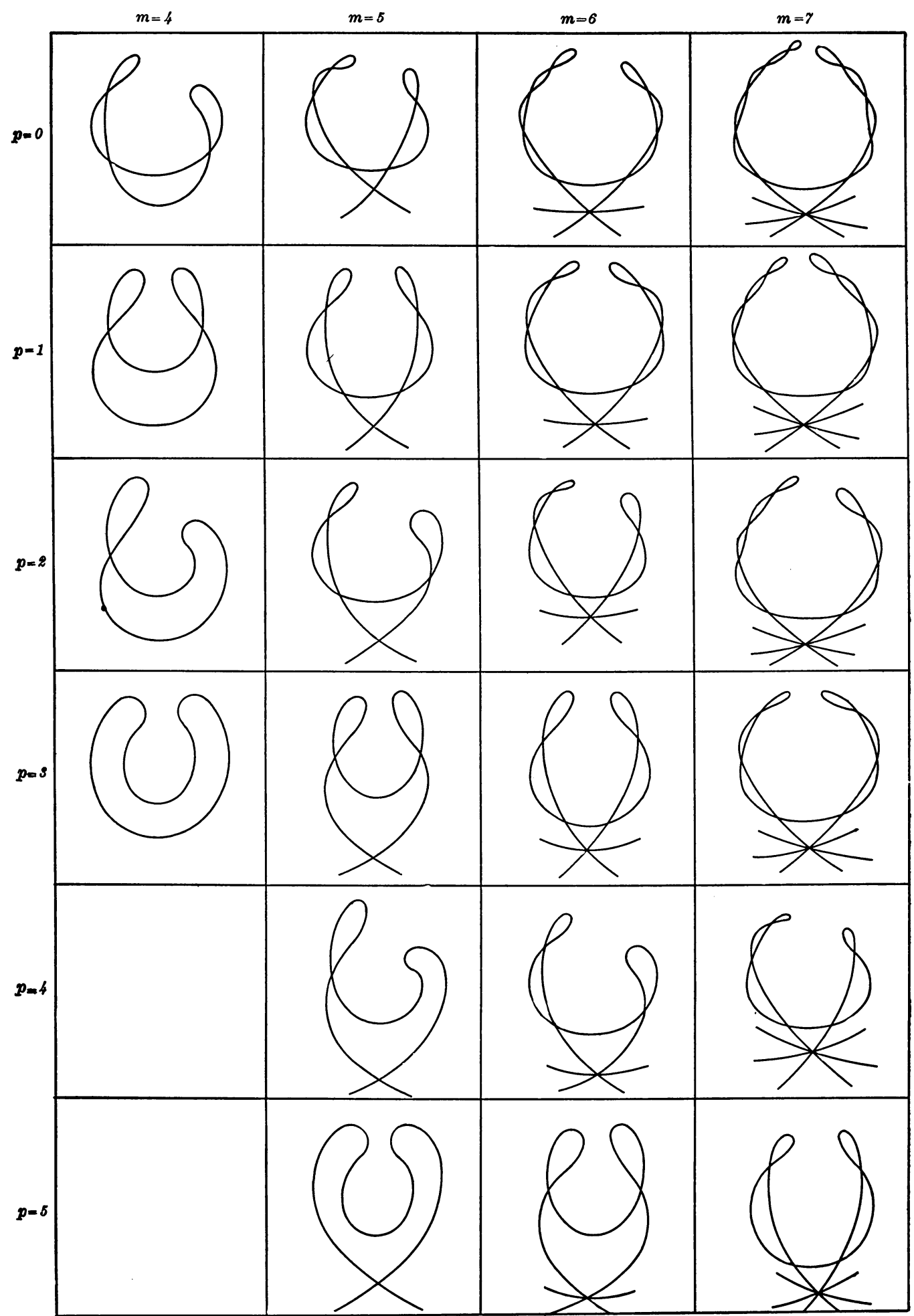

Fig. 6 . 
Any such curve is a $C_{m}$ with an $(m-3)$-point and $q$ links, where $q=2 m-5-p$. This is to be subjected to a Cremona transformation by means of curves of order $m-k$, with a fixed $(m-k-1)$-point and $2(m-k-1)$ simple points, where $2(m-k-1) \geqq 2 m-5-p$, in order that there may be a fundamental point available for every link. Hence $2 k \leqq p+3$. The fundamental points left over, in number $p+3-2 k$, can be placed on the base of the $C_{m}$; their effect is then to diminish the order of the transformed curve, for which we have the value

$$
n=m(m-k)-(m-3)(m-k-1)-(p+3-2 k)=4 m-k-p-6 \text {. }
$$

Exactly as before,

and therefore

$$
\begin{gathered}
\text { index } \geqq 2(2 m-5-p), \\
\text { index } \geqq n-(p+4-k) .
\end{gathered}
$$

The index is therefore greatest, for a given $n$ and $p$, when $k$ is as great as possible, that is, when

$$
2 k=p+3 \text { or } p+2 \text {. }
$$

(1) If $p$ be even, $2 k=p+2$;

hence

$$
\text { index } \geqq n-\frac{1}{2}(2 p+8-p-2) \geqq n-\frac{1}{2}(p+6) \text {; }
$$

$$
\text { order }- \text { index } \leqq \frac{1}{2}(p+6) \text {. }
$$

If $\frac{1}{2}(p+6)$ be even, that is, if $p=4 t-2$,

$$
\text { order }- \text { index }=2(t+1) \text {. }
$$

If $\frac{1}{2}(p+6)$ be odd, that is, if $p=4 t$,

hence

$$
\text { order }- \text { index } \leqq 2 t+3 \text {, }
$$

$$
\text { order }- \text { index }=2(t+1) \text {. }
$$

(2) Similarly if $p$ be odd, it is either of the form $4 t-1$ or of the form $4 t+1$, and in both cases,

$$
\text { order }- \text { index }=2(t+1) .
$$

Smaller values for the index, with or without a corresponding diminution in the order, can of course be obtained as before by placing some of the fundamental points on the boundaries of links, or outside the links, instead of inside.

8. In the construction of one of these complicated circuits by the linking of simpler circuits the Zeuthen circuit, derived by inversion from a simple oval, presents itself as fundamental, ranking with the simple oval and the simplest 
type of odd circuit. Two nodes are essential, and four real inflexions (Fig. 7), unless there is a cusp or another node (Fig. $7^{\prime}$ ), which obviates the necessity for two of the four inflexions. For this circuit the name "double-odd circuit" has been suggested.* Topologically, it is derivable by deformation from two odd circuits, the six real inflexions being reduced to the four actually existing by means of the extra node due to the linking of the circuits.

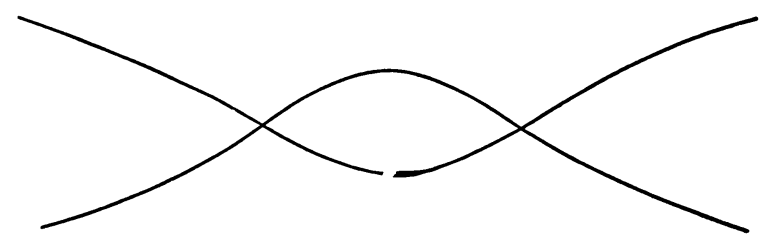

Fig. 7

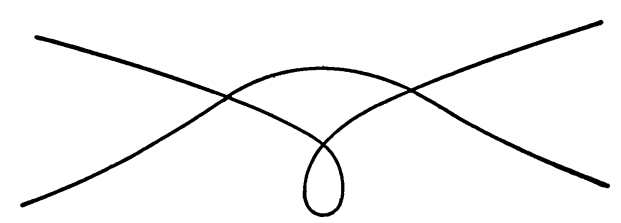

Fig. $7^{\prime}$

If $u=0$ be one of the curves of order $m$ with an $(m-3)$-point and $q$ links, of which $k$ are occupied by fundamental points in order to give rise to a circuit of index $2 k$ or $2 k+1$, then $u=\lambda$, where $\lambda$ is a small constant of proper sign, contains at least $q$ ovals, of which $k$ are occupied by fundamental points. By the Cremona transformation each of these $k$ ovals gives rise to a double-odd circuit, and the single complicated circuit, $u=0$, arises from the linking of these $k$ double-odd circuits with the circuit derived from the base of the curve. Thus the circuit of index $2 k+1$ is derived from $k$ double-odd circuits and one other; or, we may say, it is derived (topologically) from $2 k+1$ odd circuits. When 1, 2, 3 fundamental points are placed on boundaries, 1, 2, 3 of the double-odd circuits are replaced by simple odd circuits. In any case, the index of any curve here considered is equal to the number of the odd circuits from which the curve can be derived, topologically, by the process of linking.

Since each odd circuit has three real inflexions, a fact proved by Moebius, the circuit of index $i$, produced from $i$ odd circuits with the help of $i-1$ new double points if $p=1$, has at least $3 i-2(i-1)$, that is, $i+2$ real inflexions. In particular, the curve for which $i=n-2$ has at least $n$ real inflexions if $p=1$, and at least $n-2$ if $p=0$. KLeIN's equation connecting the numbers of the real singularities, namely,

${ }^{*}$ R. Gentry, 1896, On the Forms of Plane Quartic Curves. 
order + no. real inflexions +2 no. isolated tangents

$$
=\text { class }+ \text { no. real cusps }+2 \text { no. isolated points, }
$$

becomes in this case (since there cannot be an isolated tangent, which would require four imaginary intersections with a straight line)

Hence

$$
\begin{aligned}
\text { order }+ \text { no. real inflexions } & =\text { class } \\
& =2 n, \text { if } p=1, \\
& =2 n-2, \text { if } p=0 .
\end{aligned}
$$

$$
\begin{aligned}
& \text { no. real inflexions }=n \text {, if } p=1 \text {, } \\
& =n-2 \text {, if } p=0 \text {. }
\end{aligned}
$$

If however $p=0$ in virtue of an isolated point instead of an extra node on the circuit itself, the number of real inflexions $=n$.

9. Any odd circuit in the transformed plane meets the fundamental straight. lines each in an odd number of points, and therefore corresponds to a circuit passing an odd number of times through each of the simple fundamental points in the original plane. Now this circuit, passing in and out of the links, has at least $2 k$ real intersections with the $C_{m}$. Hence not only every straight line, but also every odd circuit, cuts the transformed curve in $n-2$ (in $n-2 r$ ) real points. This indicates an essential difference between the circuits here considered and the non-singular sextic circuit of index 2 , since, as has already been mentioned, there are odd circuits that do not meet this sextic. It seems therefore that it may be necessary to introduce some such term as circulation, to denote the number of odd circuits from which a given one can be derived by deformation, or, perhaps more conveniently, the minimum number of times that the circuit must be crossed in passing from an arbitrary point on the sphere to the opposite point, when the path is entirely at our disposal. This aspect of the case $I$ hope to consider at some future time; it is sufficient at present to remark that obviously

$$
\text { index }- \text { circulation } \equiv 0 \quad(\bmod 2),
$$

and that for the complicated circuits here considered

$$
\text { index }=\text { circulation } .
$$

Bryn Mawr College, Penngyluania. 